\title{
P-PET: Partial Pseudo-Exhaustive Test for High Defect Coverage
}

\author{
Abdullah Mumtaz, Michael E. Imhof, Hans-Joachim Wunderlich \\ Institute of Computer Architecture and Computer Engineering \\ University of Stuttgart, Pfaffenwaldring 47, D-70569 Stuttgart, Germany \\ email: \{mumtazah,imhof,wu\}@iti.uni-stuttgart.de
}

\begin{abstract}
Pattern generation for embedded testing often consists of a phase generating random patterns and a second phase where deterministic patterns are applied. This paper presents a method which optimizes the first phase significantly and increases the defect coverage, while reducing the number of deterministic patterns required in the second phase.

The method is based on the concept of pseudo-exhaustive testing (PET), which was proposed as a method for fault model independent testing with high defect coverage. As its test length can grow exponentially with the circuit size, an application to larger circuits is usually impractical.

In this paper, partial pseudo-exhaustive testing (P-PET) is presented as a synthesis technique for multiple polynomial feedback shift registers. It scales with actual technology and is comparable with the usual pseudo-random (PR) pattern testing regarding test costs and test application time. The advantages with respect to the defect coverage, $\mathbf{N}$-detectability for stuck-at faults and the reduction of deterministic test lengths are shown using state-of-the art industrial circuits.
\end{abstract}

Keywords-BIST, Pseudo-Exhaustive Testing, Defect Coverage, N-Detect

\section{INTRODUCTION}

Nearly three decades ago, pseudo-exhaustive testing has been proposed for increasing defect coverage without the limitations of specialized fault models [1-14]. In pseudoexhaustive testing, each output function of a combinational circuit is tested exhaustively. As in general a single output depends on a subset of all primary inputs only, testing each output function exhaustively needs much less patterns than testing the circuit exhaustively.

For a primary output $o$ of a combinational circuit $C$ with the inputs $I$ and outputs $O$, a cone is defined as the minimal subcircuit containing all structural predecessors of $o$. The set $k_{o}$ denotes all inputs connected to the output $o$, and its cardinality $\left|k_{o}\right|$ is called the cone size.

A pseudo-exhaustive test set $T$ for $C$ is a set of test patterns that includes an exhaustive test for each circuit cone. If the cones are tested in succession, the test application time is

$$
|T|=\sum_{o \in O} 2^{\left|k_{o}\right|}
$$

As some cones can be tested in parallel, the test time is limited by

$$
2^{w} \leq|T| \leq|O| \times 2^{w}
$$

where $w$ represents the largest cone size in the circuit. The inequality above shows that the test set $T$ increases exponentially with $w$. Testing the complete circuit pseudoexhaustively is only practical if $w$ does not exceed a certain limit.

The reasons why pseudo-exhaustive testing has been proposed were the limitations of fault models for reflecting real defects and for obtaining high defect coverage. With the advent of nano-electronic circuits and their problems of robustness, numerous defect mechanisms and unpredictable behavior, these reasons hold more than ever [15-18].

The reason why pseudo-exhaustive testing has not emerged as the dominating test strategy was its limitations in scaling for today's circuits. The increasing size of the maximum cones and the growing number of primary inputs and outputs made PET unfeasible, and different techniques had to be applied.

The standard approach is mixed mode testing where first pseudo-random patterns are applied, and afterwards deterministic test patterns are used for undetected faults. Pseudorandom testing can reach rather high defect coverage $[19,20]$ unless the circuit contains random pattern resistant areas.

For deterministic pattern generation, the stuck-at fault model is widely used for its simplicity, but it does not model the behavior of production defects completely [21]. Even achieving $100 \%$ stuck-at fault coverage does not guarantee the detection of all defects in a chip [22], thereby limiting the achievable defect coverage. The defect coverage can be enhanced by using the N-detect approach [20], where each single stuck-at fault is detected at least $\mathrm{N}$ times or the maximum number of times that fault can be detected. However, the size of the required deterministic test set grows significantly with the value $\mathrm{N}$ [23].

In this paper, we substitute the first phase of mixed-mode test, the pseudo-random pattern generation phase, by a novel scheme named partial pseudo-exhaustive test (P-PET). In PPET, instead of all the circuit cones, only cones up to a given size $M A X_{\text {size }}$ are tested pseudo-exhaustively. For the cones larger than $M A X_{\text {size }}$, the generated test set is not guaranteed to enumerate all of their input assignments, and behaves like pseudo-random patterns.

This scheme is based on the observation that modern circuits are well optimized for speed, and contain short paths. The resulting reduction in cone sizes means that a significant portion of the circuit can be tested pseudo-exhaustively. This implies that compared to PR testing

- testing this portion of the circuit pseudo-exhaustively results in a very high non-target fault coverage for the 
complete circuit.

- the pseudo-exhaustively tested part of the circuit offers the highest possible $N$-detectability, which significantly improves the overall defect coverage.

- a higher stuck-at fault coverage is achieved in the first phase of the mixed mode test scheme.

- the insertion of test points may further improve the fault coverage and defect coverage.

- the use of P-PET leads to a significantly lower number of deterministic pattern required in the second phase to reach a certain fault coverage.

The P-PET scheme consists of a synthesis algorithm for computing multiple feedback polynomials of a limited degree, a mapping for transforming the multiple scan chains problem to the single scan chain problem, and a multi-polynomial feedback shift register. The hardware of this register is directly taken from [24], and needs only negligible overhead compared to standard approaches. The synthesis algorithm for the multiple feedback polynomials is based on the theory published in [4], where a single polynomial of unbounded degree is computed for a single scan chain. Instead of this, we use multiple polynomials of bounded degrees, each of which covers a set of cones of a size $\leq M A X_{\text {size }}$. Dealing with all the cones $\leq M A X_{\text {size }}$ by a minimum number of polynomials is reduced to a set covering problem.

The rest of this paper is organized as follows: After describing of state of the art in the next section, the P-PET principle is presented in section III. Section IV explains the mapping of multiple scan chains to a single one, section $\mathrm{V}$ describes the used check for exhaustive enumeration, and the set covering heuristic is presented in section VI. Experiments performed on industrial circuits are discussed in section VII. We show and quantify the benefits in terms of increased fault coverage, high defect coverage, increased $\mathrm{N}$-detectability, and the reduction of the deterministic patterns additionally required.

\section{Previous work AND STATE of THE ART}

\section{A. Mixed-mode hardware schemes}

Figure 1 shows the basic embedded test architecture using multiple scan chains: Self Test Using MISR and Parallel Shift register sequence generator (STUMPS).

The pattern generation is performed by a linear feedback shift register (LFSR) connected to the scan chains. The LFSR

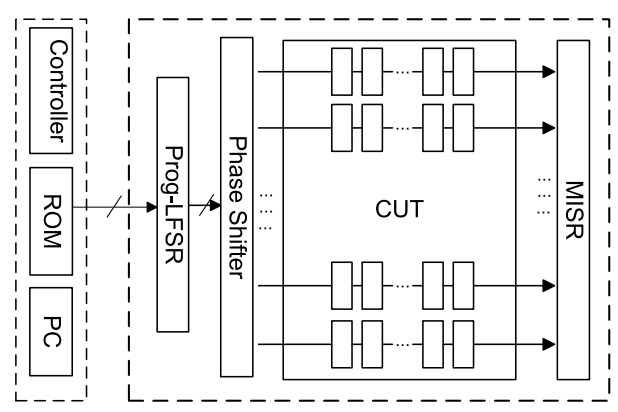

Fig. 1. Basic STUMPS Architecture. implements a fixed feedback primitive polynomial of degree $g$ and enumerates all input vectors of length $g$. In general, the pattern length $2^{g}-1$ for this is not feasible, and only a subsequence is applied during pseudo-random testing.

Due to linear dependencies, some faults may not be detected by a single LFSR-sequence. By equipping the LFSR with programmability [24], several primitive polynomials with different linear dependencies can be used while the hardware overhead consists of some AND gates (fig. 2) and the mask $\left(h_{g-1}, h_{g-2}, \ldots h_{0}\right)$.

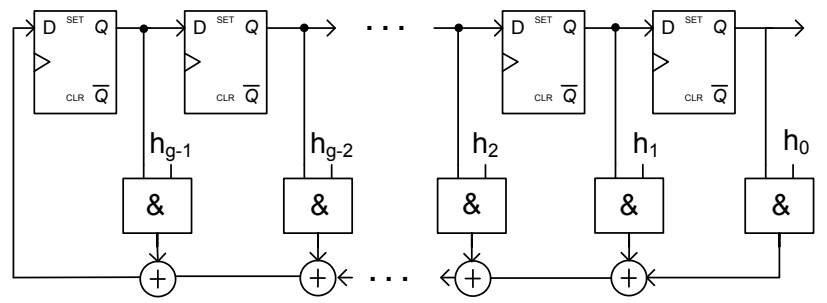

Fig. 2. Programmable LFSR [24].

The presented work adapts the programmable LFSR for the generation of pseudo-exhaustive test patterns, where the required primitive polynomials are calculated from the circuit structure. These polynomials are stored in the ROM (fig. 1) to update the programmable LFSR during pattern generation. Hence, in comparison to standard approaches (e.g. PR testing), the hardware overhead of the proposed P-PET is negligible and consist of a few AND gates and a small ROM. The approach is applicable for both embedded testing and for built-in self testing.

\section{B. Pseudo-exhaustive pattern generation}

Finding a pseudo-exhaustive test set with minimal length is known to be NP-complete [25]. However, several heuristics and techniques have been proposed to generate test sets with almost optimal sizes. Some of them include:

- Syndrome-driver counters [5].

- Constant-weight code based schemes [1].

- Hardware approaches like linear networks (XOR tree) based on linear codes [6] and linear sums [8].

- Condensed LFSRs, either based on linear codes [2] or cyclic codes [3].

- LFSR and shift register based schemes [4].

These schemes do not scale with today's circuits as they may produce test sets with extremely high pattern counts.

\section{THE P-PET PRINCIPLE}

An LFSR of length $r$ and an exhaustive LFSR sequence of length $2^{r}-1$ are uniquely defined by a characteristic polynomial or feedback polynomial $p$ of degree $r$. Let $I:=$ $\{1, \ldots, n\}$ denote the primary inputs of the combinational circuit under test. For each output $o$, we identify its cone $k_{o}=\left\{i_{1}, \ldots i_{s}\right\} \subseteq I$ with the set of its primary inputs. If the feedback polynomial is primitive, the LFSR cycles through $2^{r}-1$ different states and generates $2^{r}-1$ different patterns. 
We say, polynomial $\mathrm{p}$ tests cone $k_{o}, p \prec k_{o}$, if the $2^{r}-1$ different patterns cover all $2^{s}-1$ different assignments of $k_{o}$ except the all zero vector which will be applied separately. Let $K_{c}:=\left\{k_{o}|o \in O \wedge| k_{o} \mid \leq M A X_{\text {size }}\right\}$ be the set of all cones with $M A X_{\text {size }}$ inputs at most. Our goal is to find a minimum set $P$ of polynomials of degree $\leq M A X_{\text {size }}$, so that each cone is tested by at least one polynomial:

$$
\forall k \in K_{c} \exists p \in P \quad p \prec k
$$

The search for $P$ is organized in several steps:

1) Map the P-PET problem for multiple scan chains to the P-PET problem for a single scan chain.

2) Implement an efficient procedure for checking $p \prec k$.

3) Look for an efficient procedure to solve the NP-complete set covering problem defined by (3).

\section{P-PET FOR MULTIPLE SCAN CHAINS}

Assume the scan elements of the circuit under test are organized into $h$ scan chains, let the size of the largest scan chain be $t$. These scan chains can be treated as one big scan chain if the channel separation $t_{i}$ between the different scan chains is at least $t$ (fig. 3).

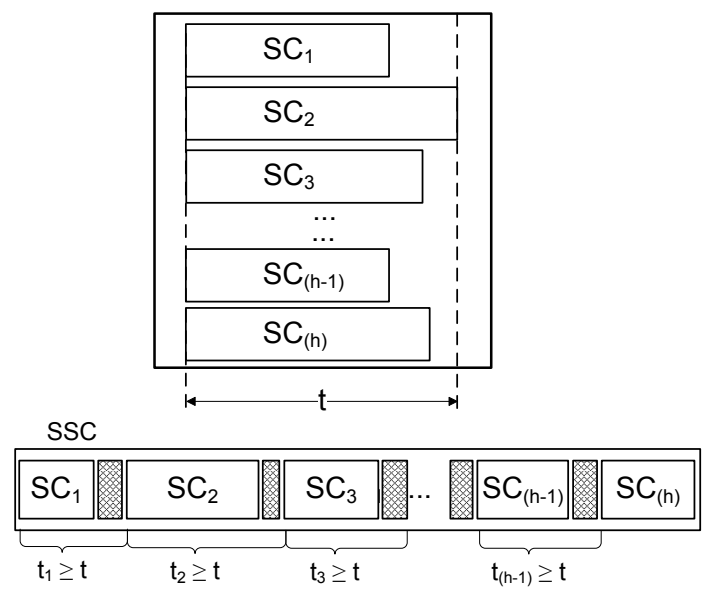

Fig. 3. Mapping of multiple scan chains

A carefully designed phase shifter [26] can be used to achieve this goal. In figure $3, h$ scan chains are mapped into a single scan chain $S S C$ where two successive scan chains $S C_{i}$ and $S C_{i+1}$ are separated by $t_{i}$. However, this cannot be directly applied to multi-polynomial LFSRs as $t_{i}$ also depends on the applied polynomial $p$. In this case, $t_{i}<t$ is possible, and a relation as seen in figure 4 may occur.

The figure contains two overlapping scanchains and presents two possible scenarios. First, if the inputs of a cone $k_{1}$ are mapped onto different positions in the unified scanchain, then $p \prec k_{1}$ is possible and has to be checked. In the second scenario, if some inputs of a cone ( $k_{2}$ in figure 4$)$ are mapped to the same positions in a virtual scan chain, these overlapping inputs will never receive different values. Hence, we can conclude without any further check that $p \prec k_{2}$ is not possible.

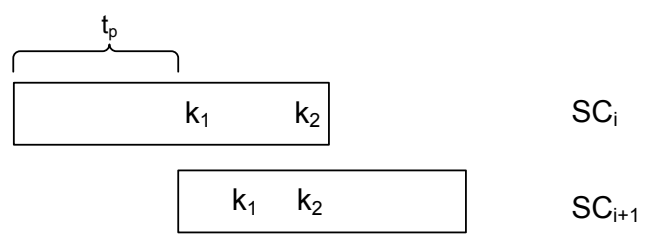

Fig. 4. Scan chain separation with and without conflict.

To distinguish the two cases, the position of each scan element $e \in S C_{i+1}$ in the virtual single scan chain is computed by

$$
\left(\sum_{j=1}^{i} t_{j}+i d(e)\right) \quad \bmod 2^{r}-1
$$

where $i d(e)$ is the index of $e$ in $S C_{i+1}$.

From now on, we assume just a virtual single scan chain.

\section{Checking For exhaustive EnUMeration}

The naive check $p \prec k$ could be implemented by generating the $2^{r}-1$ different patterns of $p$ and validating that the $2^{s}$ different assignments of $k$ are generated. However, this is extremely time consuming, and as this has to be done for a large set of polynomials it is not feasible. However, a much more efficient method is provided by Barzilai's theorem:

Theorem (Barzliai et al. [4])

Let $\left(a_{\tau}\right)_{\tau \geq 0}$ be a shift register sequence generated by a primitive feedback polynomial $p$ of degree $r$. The set $\mathrm{T}:=\left(a_{0}, \ldots, a_{r-1}\right),\left(a_{1}, \ldots, a_{r}\right), \ldots,\left(a_{2^{r}-2}, a_{0}, \ldots, a_{r-2}\right)$ is an exhaustive enumeration of the assignment of $\left(i_{1}, \ldots, i_{s}\right)$, if the remainder classes $\left(X^{i_{1}} \bmod \mathrm{p}\right), \ldots,\left(X^{i_{s}} \bmod \mathrm{p}\right)$ over $\mathrm{GF}(2)$ are linearly independent.

Figure 5 shows an example where an LFSR with feedback polynomial $p=x^{3}+x+1$ is connected to a scan chain. The polynomial is primitive and repeats the sequence after $2^{3}-1$ cycles as highlighted in the figure.

To check if a cone $k_{1}=\{0,3,4\}$, marked in the figure, receives every non zero sequence from $p$, the residue classes are calculated.

$\left(x^{0}\right) \bmod \left(x^{3}+x+1\right)=(1)$

$\left(x^{3}\right) \bmod \left(x^{3}+x+1\right)=(x+1)$

$\left(x^{4}\right) \bmod \left(x^{3}+x+1\right)=\left(x^{2}+x\right)$

As the three remainders are linearly independent, we have $p \prec k_{1}$. This can be verified by using figure 5 and initializing the LFSR to any random value. All unique $2^{3}-1$ nonzero sequences are applied at the desired positions after 11 cycles.

On the contrary, cone $k_{2}=\{0,4,5\}$ fails the linear independency check. Hence, the polynomial $p$ does not cover $k_{2}$, which can easily be verified by using figure 5 .

Barzilai's theorem reduces the check of $p \prec k$ to $s$ simple polynomial divisions and a check for linear dependency. 


\section{Set Covering Heuristic}

Let $P_{k}$ be the set of primitive polynomials of degree $k$, let $f_{p}:=\left\{k \in K_{c} / p \prec k\right\}$ be the set of all cones tested by a polynomial $p$. We want to find a set $L \subset P_{M A X_{\text {size }}}$ such that $K_{c}=\bigcup_{p \in L} f_{p}$ and $|L|$ is minimum.

The search procedure has two phases:

A) Reduction of $K_{c}$ by removing redundant cones.

B) Iterative construction of polynomials until the new $K_{c}$ is covered completely.

\section{A. Reduction of the cone set}

The bit sequence $C$, generated by an LFSR with polynomial $P(x)=\sum_{i=0}^{n} a_{i} x^{i}$ can be calculated by the following recurrence relation:

$$
y_{m+n}=a_{0} y_{m}+a_{1} y_{m+1}+\ldots+a_{n-1} y_{m+n-1}, m \geq 0
$$

For each subsequence $c=c_{0}, c_{1}, \ldots, c_{n-1}$ in $C$, there is a $c^{\prime}=c_{n-1}, c_{0}, \ldots, c_{n-2}$ where $c^{\prime}$ is a cyclic shift of $c$. This implies that in a scan chain, the position of a cone is irrelevant if the relative distances between the cone's inputs remain intact. Every displacement of this cone receives the same sequence. Therefore, we assume without loss of generality that the cones are shifted to the beginning of the scan chain, while keeping the relative distance between their inputs, and for each cone $k=\left\{i_{1}, \ldots, i_{s}\right\}$ we have $i_{1}=0$.

If we have two cones $k_{a}, k_{b}$ with $k_{a} \subseteq k_{b}$, we can remove $k_{a}$, as any enumeration of $k_{b}$ would also enumerate $k_{a}$. From now we assume that all redundant cones are removed from $K_{c}$.

Example: Suppose $K_{c}$ contains two cones $k_{2}=\{3,7,9\}$ and $k_{3}=\{12,16,18,25\}$. By shifting the cones at the beginning of the scan chain, the cones become: $k_{2}=\{0,4,6\}$ and $k_{3}=\{0,4,6,13\}$. As $k_{2} \subset k_{3}$ cone $k_{2}$ is identified as redundant and can be removed to get $K_{o c}$ with only one cone $k_{3}$.
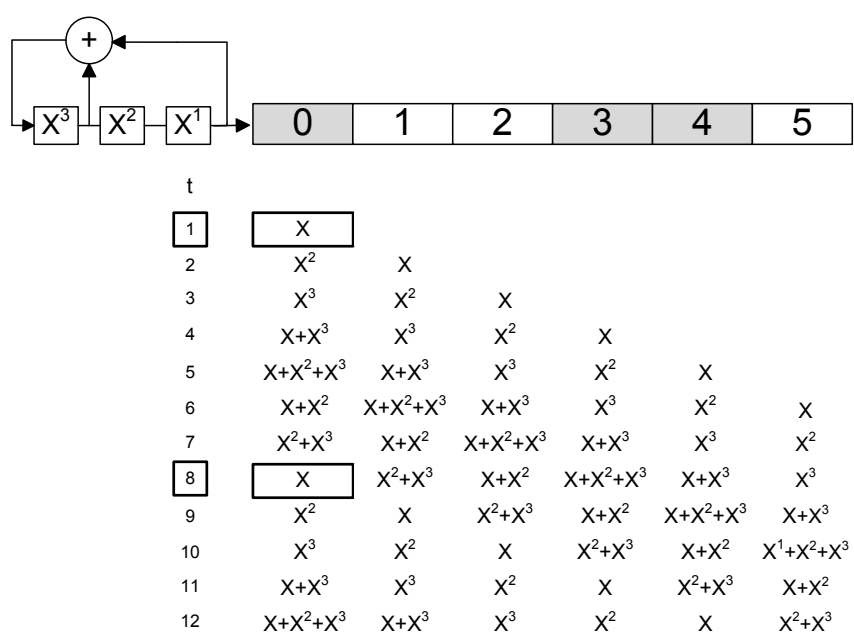

Fig. 5. LFSR sequence.

\section{B. Iterative polynomial construction}

$K_{c}$ may still contain a large number of cones $k$, and checking pairwise $p \prec k$ can be rather time consuming for all primitive polynomials of a certain degree. Hence, we check $p \prec k$ only for cones of $M A X_{\text {size }}$ and get a set of possible polynomials, each of them tests the largest number of big cones. From this set of possible polynomials, we select a polynomial which tests the largest subset of all smaller cones. The complete algorithm is found in figure 6 .

1) Let $P P:=\phi$

2) $K_{c}:=\left\{k \in K|| k \mid \leq M A X_{\text {size }}\right\}$

3) $K_{o c}:=\left\{k_{b} \in K_{c} \mid \nexists k_{a} \in K \quad k_{b} \varsubsetneqq k_{a}\right\}$

4) $M A X:=M A X_{\text {size }}$

5) $K_{d}:=\left\{k \in K_{o c}|| k \mid=M A X\right\}, i d x=M A X$

6) If $\left(K_{d}:=\phi\right) M A X=M A X-1$, goto step 5 .

7) $P_{c n d}:=\left\{\exists p \in P_{i d x} \mid \mathrm{p}\right.$ covers max cones of $\left.K_{d}\right\}$

8) If $\left(P_{c n d}:=\phi\right), i d x=i d x+1$, goto step 7 .

9) $K_{s}:=K_{o c}-K_{d}$

10) Find $p_{s} \in P_{\text {cnd }}$ that covers max cones from $K_{s}$, add $p_{s}$ to $P P$.

11) $K_{o c}:=K_{o c}-\left\{k \in K_{c} \mid\left(p_{s} \prec k\right)\right\}$

12) $K_{d}:=K_{d}-\left\{k \in K_{d} \mid\left(p_{s} \prec k\right)\right\}, P_{c n d}:=P_{c n d}-p_{s}$

13) If $\left(K_{d} \neq \phi\right)$ AND $\left(P_{c n d} \neq \phi\right)$ goto step 9

14) If $\left(K_{o c} \neq \phi\right)$ goto step 4.

15) Return $P P$.

Fig. 6. Algorithm: Calculate Covering Primitive Polynomials

The variable $M A X$ is initialized to $M A X_{\text {size }}$ and represents the largest cone size in $K_{o c}$. The set $K_{d}$ is a subset of $K_{o c}$ and contains cones of exactly the size $M A X$. If $K_{d}$ is empty, $M A X$ is decreased by one, and step 5 is repeated. A set of primitive polynomials of size $i d x\left(P_{c n d}\right)$ is found that covers a maximum number of cones from $K_{d}$. This set contains the solution candidates of the current iteration. As $\left|\mathrm{K}_{d}\right| \ll\left|\mathrm{K}_{c}\right|$, only a few cones are checked against $\mathrm{P}_{i d x}$ to get $\mathrm{P}_{c n d}$, where $\left|\mathrm{P}_{c n d}\right| \ll\left|\mathrm{P}_{i d x}\right|$. This step immensely reduces the runtime of the algorithm and makes it scalable with the circuit size.

The set $K_{s}$ contains the cones from $K_{o c}$ which are not in $K_{d}$. The polynomial $p_{s} \in P_{\text {cnd }}$ that covers a maximum number of cones from $K_{s}$ is the solution of the current iteration. The selected polynomial $\left(p_{s}\right)$ is then added to the final solution set PP. The cones which are covered by $\mathrm{p}_{s}$ are removed from $K_{o c}$ and $K_{d}$. If $K_{d}$ is not empty and there are still candidate polynomials in $P_{c n d}$, we go to step 9 . The algorithm continues unless $K_{o c}$ is empty.

The final result is a set PP containing selected primitive polynomials that covers all the cones up to the given size $M A X_{\text {size }}$.

Every polynomial in PP is used as an LFSR feedback function and all possible unique patterns per polynomial are applied. The all zero pattern is applied separately. The number of patterns to be applied are calculated by using the following equation: 


$$
\sum_{i \in P P} 2^{P D_{i}}-|P P|+1+t
$$

where $P D_{i}$ is the degree of the polynomial and the 1 represents all zero pattern.

\section{EXPERIMENTAL RESULTS}

The experiments show the advantages of P-PET with and without circuit modification by test point insertion. The experiments are performed in two major parts. First, the advantages of P-PET are proven experimentally, while the second part evaluates the additional achievable gain if circuit modifications are possible. All experiments were conducted on full scan circuits kindly provided by NXP.

\section{A. Partial Pseudo-exhaustive Testing}

1) Analysis: P The cone distribution of a circuit determines the portion being P-PET testable. Figure 7 shows this distribution for $\mathrm{p} 239 \mathrm{k}$. In both graphs, the $\mathrm{x}$-axis presents cones up to the size 32 . For example, " $<=16$ " represents all cones up to the size 16. The largest cone contained by this circuit is of size 442 . The $y$-axis in figure 7(a) represents the percentage of the cones covered. Figure 7(b) plots the percentage of gates covered by the cones up to a given size.

The results show that the majority of the cones is relatively small. More than half of the cones in the circuit p239k are of

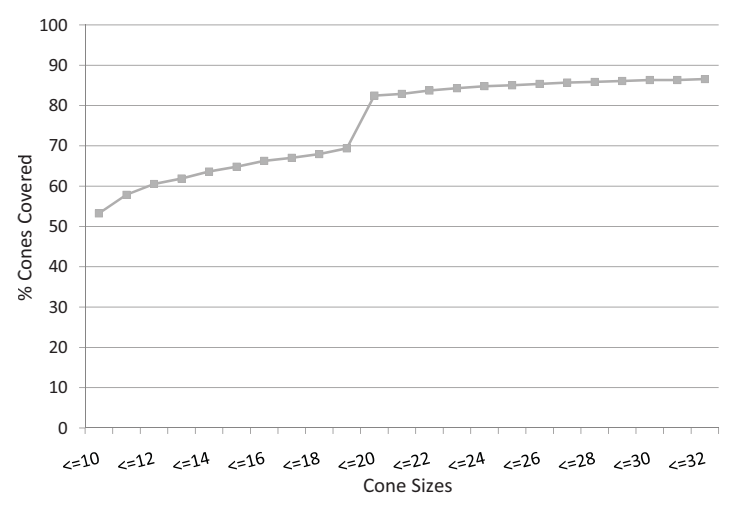

(a) Cones Sizes vs Cones Covered

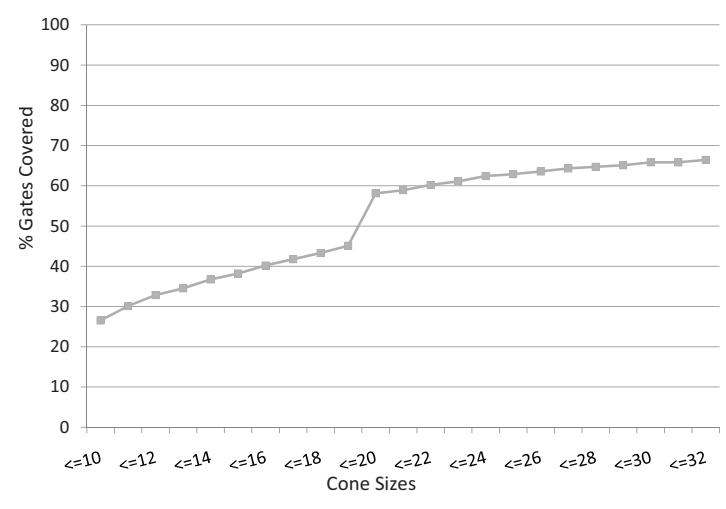

(b) Cones Sizes vs Gates Covered

Fig. 7. p239k: Achievable coverage by P-PET. size 10 or smaller. This percentage of covered cones increases to almost $85 \%$ if cones up to a size of 22 are allowed.

The portion of the circuit covered by these cones in terms of gates is presented in figure 7(b). It can be seen that the cones of limited sizes cover a significant portion of the circuit. Considering cones up to the size of 22 covers more than $60 \%$ of the circuit gates.

The maximum covered cone size $\left(M A X_{\text {size }}\right)$ is user defined, circuit dependent and test time dependent. Analyzing the cone and gate coverages of the examined circuits showed an optimal trade-off between coverage and test time at 24 , which will be used in all following experiments.

Table I presents the statistics of the considered industrial circuits. The first three columns show name, number of inputs and output (primary+pseudo-primary) of the circuits.

The name of the circuit roughly reflects the number of logic gates. The fourth column represents the percentages of the cones up to a size of 24 , while the percentages of the gates that are covered by these cones are given in the last column.

\begin{tabular}{|l|l|l|l|l|}
\hline Circuit & $\#(\mathbf{P I + P P I )}$ & $\#(\mathbf{P O + P P O )}$ & Cones $\mathbf{( \% )}$ & Gates(\%) \\
\hline p35k & 2912 & 2229 & 74.07 & 38.54 \\
\hline p45k & 3739 & 2550 & 57.28 & 55.26 \\
\hline p89k & 4632 & 4557 & 64.18 & 30.54 \\
\hline p100k & 5902 & 5829 & 82.75 & 49.76 \\
\hline p141k & 11290 & 10502 & 45.05 & 34.54 \\
\hline p239k & 18692 & 18495 & 83.91 & 62.41 \\
\hline p259k & 18713 & 18495 & 83.25 & 65.84 \\
\hline p279k & 18074 & 17827 & 58.98 & 52.16 \\
\hline p378k & 15732 & 17420 & 68.65 & 82.54 \\
\hline p418k & 30430 & 29809 & 58.42 & 48.04 \\
\hline p483k & 33264 & 32610 & 85.48 & 60.08 \\
\hline p533k & 33373 & 32610 & 83.68 & 66.66 \\
\hline
\end{tabular}

TABLE I

Cone And Gate Coverage $\left(M A X_{\text {size }}=24\right)$

For the majority of the circuits, more than $60 \%$ of the cones have a maximum size of 24 . For some circuits, this percentage is even beyond $80 \%$. The last column shows that these relatively small cones correspond to almost half of the circuit.

2) Stuck-At Fault Coverage and N-Detectability: By using the proposed synthesis scheme, we calculated the required primitive polynomials for different circuits. The calculation is performed by using a single core of a 64-bit machine running at $2.4 \mathrm{Ghz}$ with $4 \mathrm{~GB}$ RAM. Table II reports these results. The second column shows the number and degrees of the required polynomials. The third column presents the number of patterns generated from these polynomials and are calculated by using the equation 6. The time (in minutes) needed to calculate the required polynomials is shown in the last column. The numbers indicates the efficiency of the proposed heuristic, which can easily be adapted to take advantage of multi-core processors architectures to further reduce the calculation time.

For example circuit, p45k requires 1 primitive polynomial of degree 24 . The circuit p35k does not have any cone between the size 17 and 24, the largest primitive polynomial required is of degree 16 . 


\begin{tabular}{|l|l|r|r|}
\hline Circuit & Req-Poly & \#Patterns & Time(Min) \\
\hline p35k & $1 \times 2^{16}+2 \times 2^{11}$ & 72544 & 8 \\
\hline p45k & $1 \times 2^{24}$ & 16780955 & 4 \\
\hline p89k & $1 \times 2^{24}+1 \times 2^{23}$ & 25170455 & 7 \\
\hline p100k & $2 \times 2^{24}$ & 3356033 & 10 \\
\hline p141k & $2 \times 2^{24}+2 \times 2^{23}$ & 50342935 & 123 \\
\hline p239k & $3 \times 2^{24}+1 \times 2^{23}$ & 58738945 & 56 \\
\hline p259k & $3 \times 2^{24}$ & 50350359 & 178 \\
\hline p279k & $6 \times 2^{24}$ & 100681365 & 32 \\
\hline p378k & $8 \times 2^{24}$ & 134233453 & 1418 \\
\hline p418k & $5 \times 2^{24}$ & 83916506 & 115 \\
\hline p483k & $5 \times 2^{24}+1 \times 2^{23}$ & 92307947 & 325 \\
\hline p533k & $8 \times 2^{24}$ & 134251094 & 872 \\
\hline
\end{tabular}

TABLE II

Required Primitive Polynomials and Pattern Count.

Firstly, experiments are performed to compare P-PET with PR testing while considering the single stuck-at fault model. For both the cases, we apply the same number of test patterns as reported in table II. For PR we used a single primitive polynomial of degree 128. A higher degree was chosen to make the comparison realistic, as usually a primitive polynomial of a degree higher than 24 is used for PR testing. The results are presented in table III where the column "Faults" shows the total number of stuck-at faults. The number of undetected faults for P-PET and PR cases are reported under the columns "PPET" and "PR" respectively. The difference between the two approaches is calculated by using the equation

$$
\frac{P R-P P E T}{P R} \times 100
$$

and shows the percentage of extra faults which are detected by P-PET as compared to PR case. The column "Dif" reports these results. For all the circuits, P-PET shows better results than PR in detecting hard pseudo-random resistive faults. This gives P-PET a significant benefit in the second half of mixed-mode testing where much less deterministic patterns are required as compared to PR case. Circuit p378k is an exception as it does not have any pseudo-random resistive fault.

\begin{tabular}{|l|r|rrr|}
\hline & & \multicolumn{3}{|c|}{ Undetected Faults } \\
Design & Faults & PR & PPET & Dif(\%) \\
\hline p35k & 67988 & 27487 & 25476 & 7.32 \\
\hline p45k & 71848 & 288 & 189 & 34.38 \\
\hline p89k & 155794 & 14153 & 12745 & 9.95 \\
\hline p100k & 166960 & 822 & 654 & 20.44 \\
\hline p141k & 287552 & 9816 & 6735 & 31.39 \\
\hline p239k & 455992 & 7180 & 5551 & 22.69 \\
\hline p259k & 607536 & 10476 & 6024 & 42.50 \\
\hline p279k & 493744 & 24690 & 19225 & 22.13 \\
\hline p378k & 816274 & 0 & 0 & 0 \\
\hline p418k & 688808 & 44536 & 40184 & 9.78 \\
\hline p483k & 903348 & 22646 & 17669 & 21.97 \\
\hline p533k & 1148846 & 23859 & 19376 & 18.79 \\
\hline
\end{tabular}

TABLE III

Single Stuck At Fault Coverage.

In the second set of experiments, $N$-detect is used as a defect coverage metric to evaluate the effectiveness of both the approaches. We keep the same experimental setup as in the previous set of experiments except that instead of a single detect, we target to detect a fault $N=15$ times. The results are reported in table IV.

\begin{tabular}{|c|rrr|rrr|}
\hline & \multicolumn{3}{|c|}{ Undetected (\#Detect. < 15) } & \multicolumn{4}{|c|}{ Deterministic Patterns } \\
Design & PR & PPET & Dif(\%) & PR & PPET & Dif(\%) \\
\hline p35k & 32626 & 30447 & 6.67 & 17005 & 16973 & 0.19 \\
\hline p45k & 493 & 306 & 37.93 & 486 & 404 & 16.87 \\
\hline p89k & 41113 & 34316 & 16.53 & 2791 & 2357 & 15.54 \\
\hline p100k & 1246 & 922 & 26.00 & 688 & 592 & 13.95 \\
\hline p141k & 35036 & 24145 & 31.08 & 2322 & 1985 & 14.51 \\
\hline p239k & 9133 & 6181 & 32.33 & 1327 & 1037 & 21.85 \\
\hline p259k & 35128 & 22968 & 34.61 & 1462 & 1294 & 11.61 \\
\hline p279k & 53955 & 41245 & 23.55 & 7785 & 4475 & 57.48 \\
\hline p378k & 23467 & 11628 & 50.44 & 638 & 397 & 37.77 \\
\hline p418k & 62438 & 52661 & 15.65 & 2611 & 2172 & 16.81 \\
\hline p483k & 43350 & 29811 & 31.23 & 1485 & 1018 & 31.44 \\
\hline p533k & 48464 & 33317 & 31.25 & 1459 & 1193 & 18.23 \\
\hline
\end{tabular}

TABLE IV

Stuck-at Fault Coverage Targeting 15-Detect.

In the first half of the table, the faults which are either undetected or detected less than 15 times are reported for both PR and P-PET cases. The percentage of extra faults which are detected by P-PET as compared to PR are calculated by using equation 7 and are presented in the column "Dif".

The numbers show the effectiveness of the proposed P-PET patterns during N-detection. For every circuit, as compared with PR testing, it detects a significant number of extra faults which fulfills the criteria of 15-detect.

This is clearly visible in the case of circuit p378k. As reported in table III, this circuit does not have any pseudorandom resistive faults and in both test cases, all the faults are detected. However, the superiority of P-PET test patterns is evident when the two test sets are evaluated for achievable defect coverages. The $\mathrm{N}$-detect coverage of $\mathrm{PR}$ testing is much lower than with P-PET patterns, and the proposed approach detects more than $50 \%$ extra faults. These results indicate the fact that the P-PET testing not only gives advantages in detecting pseudo-random resistive faults, but is also more effective in $\mathrm{N}$-detect and achieves a high defect coverage.

Deterministic patterns were calculated which target the undetected faults in table IV by using a commercial tool. They too are reported for both the cases in table IV. The columns "PR" and "PPET" show the required number of deterministic patterns. The column "Dif" represents the difference (in percent) between the two approaches. The high N-detectability of P-PET implies that it needs significantly less deterministic patterns as compared to PR testing, and requires $0.19 \%$ to $57 \%$ less storage.

As explained before, both approaches are comparable in terms of applying the deterministic patterns. Hence, with both schemes, the deterministic patterns can be applied efficiently by using compression schemes (e.g [27, 28]). However, due to lower pattern counts, significant savings in pattern storage are achieved for the P-PET case.

3) Non-Target Faults: The quality of test sets in terms of defect coverage is further evaluated by their effect on surrogate 
(non-target) faults.

The experimental setup used previously for single stuck-at faults (table III) is retained except for the fault model. We consider four-way byzantine bridging faults as surrogate and randomly inject 40,000 faults per circuit. Their fault coverage is taken as an indicator of the un-modeled defect coverage achieved. The results are reported in table $\mathrm{V}$ where the columns 2 and 3 present the number of undetected bridging faults for P-PET and PR testing. In the last column, the percentage of the faults detected additionally by P-PET are reported.

\begin{tabular}{|l|rrr|}
\hline & \multicolumn{3}{|c}{ Un-Detected Brig faults } \\
Circuit & PR & PPET & Dif(\%) \\
\hline p35k & 17743 & 15196 & 14.35 \\
\hline p45k & 966 & 271 & 71.95 \\
\hline p89k & 4025 & 1613 & 59.93 \\
\hline p100k & 360 & 222 & 38.33 \\
\hline p141k & 934 & 380 & 59.31 \\
\hline p239k & 381 & 197 & 48.29 \\
\hline p259k & 416 & 225 & 45.91 \\
\hline p279k & 381 & 174 & 54.33 \\
\hline p378k & 601 & 141 & 76.54 \\
\hline p418k & 925 & 333 & 64.00 \\
\hline p483k & 634 & 312 & 50.79 \\
\hline p533k & 1099 & 667 & 39.31 \\
\hline
\end{tabular}

TABLE V

P-PET: NON-TARGETED BRIDGING FAULTS COVERAGE.

The P-PET test detects all bridging faults inside an exhaustively tested cone, however, bridges between two cones may not be detected. For these inter-cones bridges, it behaves like pseudo-random patterns. Compared to the application of PR patterns, this increased circuit and gate coverage achieved by P-PET significantly improves the non-target fault coverage and thereby the defect coverage. For almost all the circuits (except p35k), P-PET detects more than $38 \%$ extra faults and reaches $76 \%$ for $\mathrm{p} 378 \mathrm{k}$. These results support the previous findings of $\mathrm{N}$-detectability (table IV). As the proposed approach is more effective in detecting hard faults multiple times, it detects significantly more non-target faults.

\section{B. Pseudo-Exhaustive Testing With Circuit Modification}

When the circuit is tested pseudo-randomly, often not all of the faults are detected. One standard approach is the use of test points to improve the controllability and observability in a circuit $[29,30]$. It is a part of the standard flow, which is used by the industry and supported by major industrial tools.

Test points insertion for pseudo-exhaustive (PE) testing has been studied thoroughly [7, 10, 31]. By using test points, all of the larger cones are reduced to at most predefined maximum size, and the complete circuit is made pseudo-exhaustively testable.

We conducted experiments to investigate the impact of test point insertion on the defect coverage for both P-PET and PR testing.

For these experiments, reported in table VI, we first insert test points for PET by setting the maximum allowed cone size to 24 . Thereby it is guaranteed, that the modified circuit does not contain cones larger than size 24 . The same amount of test points are inserted for PR case and are reported in column "\#tp". Using the proposed algorithm, the required multiple primitive polynomials are found for the modified PE testable circuit. The number and degrees of the required polynomials are shown in column "Req-poly", while the fourth column presents the corresponding number of patterns. The bridging fault lists of the previous experiments (table V) are again used as surrogate faults.

In a PET modified circuit, every cone is tested pseudoexhaustively. The pseudo-random test points insertion increases the controllability and observability of the circuit but the basic property of PR testing holds and detection of all the non-target faults still remains fortuitous. These facts are reflected in the results presented in table VI. For example circuit $\mathrm{p} 279 \mathrm{k}$, the table $\mathrm{V}$ reports the difference between P-PET and PR testing as 54\%. When the test points are inserted for pseudo-exhaustive and pseudo-random testing, the difference increases to $76 \%$ (table VI).

\begin{tabular}{|l|l|c|r|rrr|}
\hline & & & \multicolumn{3}{|c|}{ \#Undet Brig faults } \\
Circuit & \#tp & Req-poly & \#Patterns & PR & PPET & Dif(\%) \\
\hline p35k & 756 & $5 \times 2^{24}$ & 83888988 & 573 & 121 & 78,88 \\
\hline p45k & 308 & $3 \times 2^{24}$ & 50335385 & 566 & 152 & 73.14 \\
\hline p89k & 2167 & $7 \times 2^{24}$ & 117445138 & 251 & 49 & 80.48 \\
\hline p100k & 1646 & $5 \times 2^{24}$ & 83891978 & 236 & 49 & 79.24 \\
\hline p141k & 2473 & $6 \times 2^{24}$ & 100674581 & 215 & 74 & 65.58 \\
\hline p239k & 3670 & $7 \times 2^{24}$ & 117459198 & 194 & 96 & 50.52 \\
\hline p259k & 4007 & $8 \times 2^{24}$ & 134236434 & 220 & 101 & 54.09 \\
\hline p279k & 4662 & $7 \times 2^{24}$ & 117458580 & 125 & 29 & 76.80 \\
\hline p378k & 1820 & $8 \times 2^{24}$ & 134233453 & 534 & 85 & 84.08 \\
\hline p418k & 4581 & $9 \times 2^{24}$ & 151028200 & 378 & 112 & 70.37 \\
\hline p483k & 6887 & $9 \times 2^{24}$ & 151025366 & 402 & 60 & 85.07 \\
\hline p533k & 7463 & $11 \times 2^{24}$ & 184582739 & 414 & 89 & 78.50 \\
\hline
\end{tabular}

TABLE VI

REQuired Polynomials AND NON-TARGETED BRIDGING FAUlts COVERAgE WITH TEST POINTS INSERTION.

This is true for all the circuits and shows that the insertion of test points for pseudo-exhaustive testing has greater impact on non-target faults and defect coverage than doing it for pseudorandom case.

\section{CONCLUSION}

We introduced a new approach called partial pseudoexhaustive test (P-PET), where cones up to a given size are tested pseudo-exhaustively. A synthesis technique for multiple polynomial feedback shift registers is presented which scales with actual technology and is comparable with the usual pseudo-random pattern testing regarding test costs and test application time.

With state-of-the art industrial circuits, we showed and quantified the advantages of the proposed scheme. The defect coverage was evaluated by using non-target fault and $\mathrm{N}$ detectability metrics and the results show the substancial advantages achieved by P-PET. We also showed that the use 
of P-PET results in a major reduction in the number of deterministic pattern required to reach a certain fault coverage.

The defect coverage can be further improved if circuit modifications are possible. We showed that the insertion of pseudo-exhaustive test points yields in significant benefits if compared to circuit modifications for the pseudo-random case.

\section{ACKNOWLEDGEMENT}

The authors would like to thank NXP for providing the industrial circuits.

\section{REFERENCES}

[1] E. McCluskey, "Verification Testing - A Pseudoexhaustive Test Technique," Proc. of IEEE Transactions on Computers, pp. 541$546,1984$.

[2] L. Wang and E. McCluskey, "Condensed Linear Feedback Shift Register (LFSR) Testing A Pseudoexhaustive Test Technique," Proc. of IEEE transactions on computers, vol. 35, no. 4, pp. 367-370, 1986.

[3] _ - "Linear feedback shift register design using cyclic codes," Proc. of IEEE Transactions on Computers, vol. 37, no. 10, pp. 1302-1306, 1988.

[4] Z. Barzilai, D. Coppersmith, and A. Rosenberg, "Exhaustive generation of bit patterns with applications to VLSI selftesting," Proc. of IEEE Transactions on Computers, vol. 100, no. 32, pp. 190-194, 1983.

[5] Z. Barzilai, J. Savir, G. Markowsky, and M. Smith, "The weighted syndrome sums approach to VLSI testing," Proc. of IEEE Transactions on Computers, pp. 996-1000, 1981.

[6] N. Vasanthavada and P. Marinos, "An operationally efficient scheme for exhaustive test-pattern generation using linear codes," in Proc. of IEEE International Test Conference, 1985, pp. 476-482.

[7] I. Shperling and E. McCluskey, "Circuit segmentation for pseudo-exhaustive testing via simulated annealing," in Proc. of IEEE International Test Conference, 1987, pp. 58-65.

[8] S. Akers, "On the use of linear sums in exhaustive testing," in Proc. of International Symposium on Fault-Tolerant Computing, 1985, pp. 148-153.

[9] S. Hellebrand, H. Wunderlich, and O. Haberl, "Generating Pseudo-Exhaustive Vectors for External Testing," in Proc. of IEEE International Test Conference, 1990, pp. 670-679.

[10] S. Hellebrand and H. Wunderlich, "Tools and devices supporting the pseudo-exhaustive test," in Proc. of Conference on European Design Automation, 1990, pp. 13-17.

[11] D. Tang and L. Woo, "Exhaustive test pattern generation with constant weight vectors," Proc. of IEEE Transactions on Computers, pp. 1145-1150, 1983.

[12] J. Udell Jr, "Test set generation for pseudo-exhaustive BIST," in Proc. of International Conference on Computer Aided Design, 1986, pp. 52-55.

[13] H. Wunderlich and S. Hellebrand, "Generating pattern sequences for the pseudo-exhaustive test of MOS-circuits," Proc. of International Symposium on Fault-Tolerant Computing, pp. 36-41, 1988.

[14] — "The pseudo-exhaustive test of sequential circuits," in Proc. of IEEE International Test Conference, 1989, pp. 19-27.
[15] R. Aitken, "Nanometer technology effects on fault models for IC testing," Proc. of IEEE Computer society press, vol. 32, no. 11 , pp. 46-51, 2002.

[16] J. McPherson, "Reliability challenges for $45 \mathrm{~nm}$ and beyond," in Proc. of ACM/IEEE Design Automation Conference, 2006, pp. 176-181.

[17] F. Hopsch, B. Becker, S. Hellebrand, I. Polian, B. Straube, W. Vermeiren, and H.-J. Wunderlich, "Variation-Aware Fault Modeling," in Proc. of IEEE Asian Test Symposium, 2010, pp. 87-93.

[18] B. Becker, S. Hellebrand, I. Polian, B. Straube, W. Vermeiren, and $\mathrm{H}$. Wunderlich, "Massive statistical process variations: A grand challenge for testing nanoelectronic circuits," in Proc. of International Conference on Dependable Systems and Networks Workshops, 2010, pp. 95-100.

[19] C. Tseng, S. Mitra, E. McCluskey, and S. Davidson, "An evaluation of pseudo random testing for detecting real defects," in Proc. of IEEE VLSI Test Symposium, 2001, pp. 404-409.

[20] S. Ma, P. Franco, and E. McCluskey, "An experimental chip to evaluate test techniques: Experiment results," in Proc. of IEEE International Test Conference, 1995, pp. 663-672.

[21] E. McCluskey and C. Tseng, "Stuck-fault tests vs. actual defects," in Proc. of IEEE International Test Conference, 2000, pp. 336-343.

[22] L. Wang, M. Mercer, T. Williams, and S. Kao, "On the decline of testing efficiency as fault coverage approaches $100 \%$," in Proc. of IEEE VLSI Test Symposium, 1995, pp. 74-83.

[23] I. Pomeranz and S. Reddy, "Stuck-at tuple-detection: A fault model based on stuck-at faults for improved defect coverage," in Proc. of IEEE VLSI Test Symposium, 2002, pp. 289-294.

[24] S. Hellebrand, S. Tarnick, J. Rajski, and B. Courtois, "Generation of vector patterns through reseeding of multiple-polynomial linear feedback shift registers," in Proc. of IEEE International Test Conference, 1992, pp. 120-129.

[25] G. Seroussi and N. Bshouty, "Vector sets for exhaustive testing of logic circuits," Proc. of IEEE Transactions on Information Theory, vol. 34, no. 3, pp. 513-522, 1988

[26] J. Rajski, N. Tamarapalli, and J. Tyszer, "Automated synthesis of phase shifters for built-in self-test applications," Proc. of IEEE Transactions on Computer-Aided Design of Integrated Circuits and Systems, vol. 19, no. 10, pp. 1175-1188, 2002.

[27] A. Hakmi, S. Holst, H. Wunderlich, J. Schlöffel, F. Hapke, and A. Glowatz, "Restrict encoding for mixed-mode BIST," in Proc. of IEEE VLSI Test Symposium, 2009, pp. 179-184.

[28] L. Li, K. Chakrabarty, and N. Touba, "Test data compression using dictionaries with selective entries and fixed-length indices," Proc. of ACM Transactions on Design Automation of Electronic Systems, vol. 8, no. 4, pp. 470-490, 2003.

[29] N. Tamarapalli and J. Rajski, "Constructive Multi Phase Test Point Insertion for Scan-based BIST," in Proc. of IEEE International Test Conference., 1996, pp. 649-658.

[30] Y. Savaria, M. Youssef, B. Kaminska, and M. Koudil, "Automatic test point insertion for pseudo-random testing," in Proc. of IEEE International Symposium on Circuits and Systems., 1991, pp. 1960-1963.

[31] R. Srinivasan, S. Gupta, and M. Breuer, "An efficient partitioning strategy for pseudo-exhaustive testing," in Proc. of International Design Automation Conference, 1993, pp. 242248. 